\title{
Expressiveness, Simplicity, and Users
}

\author{
Craig Chambers
}

Google

\begin{abstract}
I have worked on several different language design and optimizing compiler projects, and I am often surprised by which ideas turn out to be the most successful. Oftentimes it is the simplest ideas that seem to get the most traction in the larger research or user community and therefore have the greatest impact. Ideas I might consider the most sophisticated and advanced can be challenging to communicate, leading to less influence and adoption. This effect is particularly pronounced when seeking to gain adoption among actual users, as opposed to other researchers. In this talk I will discuss examples of the tradeoffs among sophistication, simplicity, and impact in my previous research work in academia and in my current work at Google.
\end{abstract}

